\title{
Finite-Horizon Reliable Control with Randomly Occurring Uncertainties and Nonlinearities Subject to Output Quantization *
}

\author{
Hongli Dong a,b,*, Zidong Wang ${ }^{\mathrm{c}}$, Steven X. Ding ${ }^{\mathrm{b}}$, Huijun Gao ${ }^{\mathrm{d}}$ \\ ${ }^{a}$ College of Electrical and Information Engineering, Northeast Petroleum University, Daqing 163318, China. \\ ${ }^{\mathrm{b}}$ Institute for Automatic Control and Complex Systems, University of Duisburg-Essen, 4705\%, Germany. \\ ${ }^{\mathrm{c}}$ Department of Computer Science, Brunel University, Uxbridge, Middlesex, UB8 3PH, U.K. \\ ${ }^{\mathrm{d}}$ Research Institute of Intelligent Control and Systems, Harbin Institute of Technology, Harbin 150001, China.
}

\begin{abstract}
This paper deals with the finite-horizon reliable $\mathcal{H}_{\infty}$ output feedback control problem for a class of discrete time-varying systems with randomly occurring uncertainties (ROUs), randomly occurring nonlinearities (RONs) as well as measurement quantizations. Both the deterministic actuator failures and probabilistic sensor failures are considered in order to reflect the reality. The actuator failure is quantified by a deterministic variable varying in a given interval and the sensor failure is governed by an individual random variable taking value on $[0,1]$. Both the nonlinearities and the uncertainties enter into the system in random ways according to Bernoulli distributed white sequences with known conditional probabilities. The main purpose of the problem addressed is to design a time-varying output feedback controller over a given finite horizon such that, in the simultaneous presence of ROUs, RONs, actuator and sensor failures as well as measurement quantizations, the closedloop system achieves a prescribed performance level in terms of the $\mathcal{H}_{\infty}$-norm. Sufficient conditions are first established for the robust $\mathcal{H}_{\infty}$ performance through intensive stochastic analysis, and then a recursive linear matrix inequality approach is employed to design the desired output feedback controller achieving the prescribed $\mathcal{H}_{\infty}$ disturbance rejection level. A numerical example is given to demonstrate the effectiveness of the proposed design scheme.
\end{abstract}

Key words: Finite-horizon reliable control; Time-varying systems; Actuator and sensor failure; Randomly occurring uncertainties; Randomly occurring nonlinearities.

\section{Introduction}

For several decades, stochastic control and nonlinear control serve as two of the most active research areas in systems and control that have found successful applications in a variety of engineering systems such as automotive engines, robot manipulators, aircraft and electrical motors. So far, considerable research attention has been devoted to the theoretical research on control problems for nonlinear stochastic systems, see $[1,3,5,6,9,11,20$, $22,24,30]$ and the references therein. For example, for different kinds of nonlinear stochastic systems, the $\mathcal{H}_{\infty}$ output feedback control problem has been investigated in [24], the adaptive fuzzy control problem has been proposed in [22], the neural-network-based controller design has been addressed in [23], the adaptive sliding

* This work was supported in part by the National Natural Science Foundation of China under Grants 61329301, 61134009, 61273156, 61333012, 61422301 and 61374127, the Scientific and Technology Research Foundation of Heilongjiang Education Department of China under Grant 12541061, the Engineering and Physical Sciences Research Council (EPSRC) of the U.K., the Royal Society of the U.K., and the Alexander von Humboldt Foundation of Germany.

* Corresponding author. Tel.: +86 459 6503373; fax: +86 4596503373.

Email addresses: shiningdhl@gmail.com (Hongli Dong), Zidong.Wang@brunel.ac.uk (Zidong Wang). mode controller has been designed in $[4,10,17,18]$ and the observer-based control problems have been solved in [25], respectively. Among various descriptions of nonlinearities, the so-called randomly occurring nonlinearities (RONs) [26] cater for those randomly changeable nonlinearities in terms of their types and/or intensities governed by stochastic variables. RONs, which typically occur in networked environments, encompass several well-studied nonlinearities in stochastic systems and have thus stirred particular research interests in the past few years.

It is noticeable that, in almost all the aforementioned literature, the components of the control systems have been implicitly assumed to be fully reliable. This assumption is, unfortunately, not always true since the failures of control components (e.g. sensors and actuators) often occur in practical applications due to a variety of reasons, for example, the abrupt changes of working conditions, the erosion caused by sever circumstance, the internal component constraints, the intense external disturbance and the aging of sensors or actuators, etc. Therefore, it is of both practical significance and theoretical importance to design a reliable controller against possible actuator and sensor failures such that the essential performance of the controlled system can be guaranteed $[13,19]$. In fact, in the past two decades, the problem of reliable controller design has attracted much research attention and many approaches have been proposed in the literature 
including Hamilton-Jacobi equation approach [14, 15], robust pole region assignment technique [8], algebraic Riccati equation approach $[21,29]$ and linear matrix inequality approach $[12,16,27]$. Despite the fruitful results on time-invariant systems over an infinite horizon, it is worth pointing out that the finite-horizon reliable control problem for time-varying systems has not been thoroughly investigated yet, not to mention the case complicated further by nonlinearity and stochasticity.

As is well known, modeling errors (usually parameter uncertainties) constitute an important kind of complexities for system modeling that has a great impact on the subsequent system analysis and design [28]. In today's networked systems, it is quite common that the network load is randomly fluctuated and the signal transmission suffers from unpredictable networked-induced phenomena owing to limited bandwidth. As such, the occurrence of the parameter uncertainties in a networked environment is often of random nature resulting from abrupt phenomena such as modification of the operating point of a linearized model of nonlinear systems, random failures and repairs of the components, changes in the interconnections of subsystems and sudden environment disturbances, etc. Very recently, in [10], the concept of randomly occurring uncertainties (ROUs) has been introduced to reflect the probabilistic fashion of the networkinduced modeling error and the corresponding sliding mode control problem has been considered. However, when it comes to the finite-horizon reliable $\mathcal{H}_{\infty}$ control problem involving ROUs for nonlinear time-varying stochastic systems, the related results are very few and the situation is even worse when measurement quantizations, actuator and sensor failures are also taken into account. It is, therefore, the aim of this paper to shorten such a gap by addressing the finite-horizon reliable $\mathcal{H}_{\infty}$ control problem with presence of ROUs, RONs as well as measurement quantizations.

Motivated by the above discussion, in this paper, we launch a major study on the finite-horizon reliable $\mathcal{H}_{\infty}$ output-feedback control problem for a class of discrete time-varying stochastic systems with simultaneous presence of actuator and sensor failures, ROUs, RONs and measurement quantizations. Some sufficient conditions are established, via intensive stochastic analysis, to guarantee the existence of the desired time-varying output feedback controller gains, and then such controller gains are characterized by solving a set of recursive matrix inequalities. A simulation example is finally presented to illustrate the effectiveness of the proposed design scheme. The main contributions of this paper are highlighted as follows. 1) The problem addressed is new in the sense that this paper represents the first of few attempts to deal with the finite-horizon reliable $\mathcal{H}_{\infty}$ output feedback control problem against actuator and sensor failures for a class of discrete time-varying stochastic systems. 2) The system under consideration is comprehensive to cover time-varying parameters, actuator and sensor failures, ROUs, RONs and measurement quantizations, hence reflecting the reality more closely. 3) The algorithm developed is computationally appealing in terms of its recursive nature suitable for online application.

The rest of this paper is outlined as follows. In Section 2 , a class of discrete time-varying stochastic systems is presented with actuator and sensor failures, ROUs,
RONs and measurement quantizations. The problem under consideration is formulated. In Section 3 , by employing the stochastic analysis techniques, some sufficient conditions are established to guarantee the desired output feedback controller performances and then the controller gains are obtained by solving a set of recursive matrix inequalities. A simulation example is given in Section 4 to demonstrate the main results obtained. Finally, we conclude the paper in Section 5.

Notation. The notation used here is standard except where otherwise stated. $\mathbb{R}^{n}$ and $\mathbb{R}^{n \times m}$ denote, respectively, the $n$-dimensional Euclidean space and the set of all $n \times m$ real matrices. $l[0, N]$ is the space of vector functions over $[0, N]$. The notation $X \geq Y$ (respectively, $X>Y$ ), where $X$ and $Y$ are real symmetric matrices, means that $X-Y$ is positive semi-definite (respectively, positive definite). $M^{T}$ represents the transpose of the matrix $M .0$ represents zero matrix of compatible dimensions. The $n$-dimensional identity matrix is denoted as $I_{n}$ or simply $I$, if no confusion is caused. $\operatorname{diag}\{\cdots\}$ stands for a block-diagonal matrix. $\mathbb{E}\{x\}$ and $\mathbb{E}\{x \mid y\}$ will, respectively, denote expectation of the stochastic variable $x$ and expectation of $x$ conditional on $y$. Prob $\{\cdot\}$ means the occurrence probability of the event ".". In symmetric block matrices, "*" is used as an ellipsis for terms induced by symmetry. The symbol $\otimes$ denotes the Kronecker product. Matrices, if they are not explicitly specified, are assumed to have compatible dimensions.

\section{Problem Formulation}

Consider the following uncertain discrete time-varying nonlinear stochastic system defined on $k \in[0, N]$ :

$$
\left\{\begin{aligned}
x(k+1)= & (A(k)+\alpha(k) \Delta A(k)) x(k) \\
& +B_{1}(k) u(k)+\beta(k) g(k, x(k)) \\
& +D(k) w(k) \\
z(k)= & M(k) x(k)+B_{2}(k) u(k) \\
x(0)= & \varphi_{0}
\end{aligned}\right.
$$

where $x(k) \in \mathbb{R}^{n_{x}}$ represents the state vector; $u(k) \in$ $\mathbb{R}^{n_{u}}$ is the control input; $z(k) \in \mathbb{R}^{n_{z}}$ is the controlled output; $w(k) \in \mathbb{R}^{n_{w}}$ is the disturbance input which belongs to $l[0, N]$; and $\varphi_{0}$ is a given real initial value. $A(k)$, $B_{1}(k), B_{2}(k), D(k)$ and $M(k)$ are known, real, timevarying matrices with appropriate dimensions.

The nonlinear function $g(k, x(k))$ satisfies the following condition:

$$
\|g(k, x(k))\|^{2} \leq \varepsilon(k)\|E(k) x(k)\|^{2}
$$

where $\varepsilon(k)>0$ is a known positive scalar and $E(k)$ is a known time-varying matrix.

The real-valued matrix $\Delta A(k)$ represents the normbounded parameter uncertainties of the following structure

$$
\Delta A(k)=H_{a}(k) F(k) N(k),
$$


where $H_{a}(k)$ and $N(k)$ are known time-varying matrices, while $F(k)$ is an unknown time-varying matrix function satisfying the following condition

$$
F^{T}(k) F(k) \leq I .
$$

The stochastic variables $\alpha(k) \in \mathbb{R}$ and $\beta(k) \in \mathbb{R}$ are Bernoulli distributed white sequences taking values on either 0 or 1 with

$\operatorname{Prob}\{\alpha(k)=1\}=\bar{\alpha}, \quad \operatorname{Prob}\{\alpha(k)=0\}=1-\bar{\alpha}$,

$\operatorname{Prob}\{\beta(k)=1\}=\bar{\beta}, \quad \operatorname{Prob}\{\beta(k)=0\}=1-\bar{\beta}$,

where $\bar{\alpha} \in[0,1]$ and $\bar{\beta} \in[0,1]$ are known constants.

Remark 1 The random variables $\alpha(k)$ and $\beta(k)$ are introduced to characterize the phenomena of the ROUs and the RONs, respectively. The uncertainty $\Delta A$ and the nonlinearity $g$ enter into the system in random ways according to individual Bernoulli distributions. Such a statistics description is particularly suitable for reflecting parameter/nonlinear variations that are unpredictable but appear in a random way with known probability laws.

In this paper, the measurement $\bar{y}(k)$ with probabilistic sensor failures is described by

$$
\begin{aligned}
\bar{y}(k) & =\Xi(k) C(k) x(k)+\bar{E}(k) v(k) \\
& =\sum_{j=1}^{n_{y}} \rho_{j}(k) C_{j}(k) x(k)+\bar{E}(k) v(k)
\end{aligned}
$$

where $v(k) \in \mathbb{R}^{n_{v}}$ is the measure noise belonging to $l[0, N] . \Xi(k)$ is defined as $\Xi(k):=\operatorname{diag}\left\{\rho_{1}(k), \ldots, \rho_{n_{y}}(k)\right\}$ where $\rho_{j}(k)$ has the probability density function $\zeta_{j}(s)$ $\left(j=1, \ldots, n_{y}\right)$ on the interval $[0,1]$ with mathematical expectation $\bar{\mu}_{j}$ and variance $\chi_{j}^{2} . C_{j}(k)$ is defined by

$$
C_{j}(k):=\operatorname{diag}\{\underbrace{0, \cdots, 0}_{j-1}, 1, \underbrace{0, \cdots, 0}_{n_{y}-j}\} C(k) .
$$

In the sequel, we denote $\bar{\Xi}=\mathbb{E}\{\Xi(k)\}=\operatorname{diag}\left\{\bar{\mu}_{1}, \bar{\mu}_{2}, \ldots\right.$, $\left.\bar{\mu}_{n_{y}}\right\}$. Throughout the paper, we assume that $\alpha(k), \beta(k)$ and $\rho_{j}(k)\left(j=1, \ldots, n_{y}\right)$ are unrelated random variables.

In a networked environment, it is often the case that $\bar{y}(k)$ is quantized before being transmitted to the controller. Let us denote the quantizer as $q(\cdot)=\left[\begin{array}{llll}q_{1}(\cdot) & q_{2}(\cdot) \cdots & q_{n_{y}}(\cdot)\end{array}\right]^{T}$ which is symmetric, i.e., $q_{j}(-v)=-q_{j}(v)\left(j=1, \ldots, n_{y}\right)$. The map of the quantization process is

$$
y(k)=\left[q_{1}\left(\bar{y}^{(1)}(k)\right) q_{2}\left(\bar{y}^{(2)}(k)\right) \cdots q_{n_{y}}\left(\bar{y}^{\left(n_{y}\right)}(k)\right)\right]^{T}
$$

where $y(k) \in \mathbb{R}^{n_{y}}$ and $\bar{y}^{(i)}(k)\left(i=1, \ldots, n_{y}\right)$ denotes the $i$ th element of $\bar{y}(k)$. For each $q_{j}(\cdot)(1 \leq j \leq m)$, the set of quantization levels is described by

$$
\begin{array}{r}
\mathscr{U}_{j}=\left\{ \pm \hat{\mu}_{i}^{(j)}, \hat{\mu}_{i}^{(j)}=\bar{\chi}_{j}^{i} \hat{\mu}_{0}^{(j)}, i=0, \pm 1, \pm 2, \cdots\right\} \cup\{0\} \\
0<\bar{\chi}_{j}<1, \hat{\mu}_{0}^{(j)}>0
\end{array}
$$

and each of the quantization level corresponds to a segment such that the quantizer maps the whole segment to this quantization level.

According to [7], the logarithmic quantizer is given by

$q_{j}\left(\bar{y}^{(j)}(k)\right)=\left\{\begin{array}{cc}\hat{\mu}_{i}^{(j)}, \frac{1}{1+\delta_{j}} \hat{\mu}_{i}^{(j)} \leq \bar{y}^{(j)}(k) \leq \frac{1}{1-\delta_{j}} \hat{\mu}_{i}^{(j)} \\ 0, & \bar{y}^{(j)}(k)=0 \\ -h_{j}\left(-\bar{y}^{(j)}(k)\right), & \bar{y}^{(j)}(k)<0\end{array}\right.$

where $\delta_{j}=\left(1-\bar{\chi}_{j}\right) /\left(1+\bar{\chi}_{j}\right)$. It can be easily seen from the above definition that $q_{j}\left(\bar{y}^{(j)}(k)\right)=\left(1+\Delta_{k}^{(j)}\right) \bar{y}^{(j)}(k)$ with $\left|\Delta_{k}^{(j)}\right| \leq \delta_{j}$.

Defining $\Delta_{k}:=\operatorname{diag}\left\{\Delta_{k}^{(1)}, \ldots, \Delta_{k}^{\left(n_{y}\right)}\right\}$, the measurements with quantization effect and sensor failures can be expressed as

$$
y(k)=\left(I+\Delta_{k}\right) \bar{y}(k) .
$$

Furthermore, by defining $\bar{\Delta}:=\operatorname{diag}\left\{\delta_{1}, \cdots, \delta_{n_{y}}\right\}$ and $\bar{F}(k):=\Delta_{k} \bar{\Delta}^{-1}$, we can see that $\bar{F}(k)$ is an unknown real-valued time-varying matrix satisfying $\bar{F}(k) \bar{F}^{T}(k) \leq$ I.

When the actuators experience failures, we use $u^{F}(k)$ to describe the control signal sent from actuators and the control input of actuator fault can therefore be described as follows:

$$
u^{F}(k)=G(k) u(k)
$$

where $G(k)$ is the actuator fault matrix with

$$
\left\{\begin{array}{l}
G(k)=\operatorname{diag}\left\{g_{1}(k), \ldots, g_{n_{u}}(k)\right\} \\
\underline{G}(k) \leq G(k) \leq \bar{G}(k) \\
\underline{G}(k)=\operatorname{diag}\left\{\underline{g}_{1}(k), \ldots, \underline{g}_{n_{u}}(k)\right\} \geq 0 \\
\bar{G}(k)=\operatorname{diag}\left\{\bar{g}_{1}(k), \ldots, \bar{g}_{n_{u}}(k)\right\} \leq I
\end{array}\right.
$$

and the variables $g_{i}(k)\left(i=1,2, \ldots, n_{u}\right)$ quantify the failures of the actuators. $g_{i}(k)$ and $\bar{g}_{i}(k)$ serve as the lower and the upper bounds on $g_{i}(k)$, respectively. 
Defining

$$
\begin{aligned}
G_{0}(k) & =\operatorname{diag}\left\{\hat{g}_{1}(k), \ldots, \hat{g}_{n_{u}}\right\}:=\frac{\underline{G}(k)+\bar{G}(k)}{2} \\
& =\operatorname{diag}\left\{\frac{\underline{g}_{1}(k)+\bar{g}_{1}(k)}{2}, \ldots, \frac{\underline{g}_{n_{u}}(k)+\bar{g}_{n_{u}}(k)}{2}\right\} \\
\tilde{G}(k) & =\operatorname{diag}\left\{\tilde{g}_{1}(k), \ldots, \tilde{g}_{n_{u}}(k)\right\}:=\frac{\bar{G}(k)-\underline{G}(k)}{2} \\
& =\operatorname{diag}\left\{\frac{\bar{g}_{1}(k)-\underline{g}_{1}(k)}{2}, \ldots, \frac{\bar{g}_{n_{u}}(k)-\underline{g}_{n_{u}}(k)}{2}\right\}(1
\end{aligned}
$$

the matrix $G(k)$ can be rewritten as

$$
\begin{aligned}
G(k) & =G_{0}(k)+\Delta_{G}(k) \\
& =G_{0}(k)+\operatorname{diag}\left\{\tau_{1}(k), \ldots, \tau_{n_{u}}(k)\right\}
\end{aligned}
$$

where $\tau_{i}(k)\left(i=1,2, \ldots, n_{u}\right)$ are certain scalars satisfy$\operatorname{ing}\left|\tau_{i}(k)\right| \leq \tilde{g}_{i}(k)$.

In this paper, we adopt the following time-varying output-feedback controller for system (1):

$$
\left\{\begin{array}{l}
x_{c}(k+1)=A_{c}(k) x_{c}(k)+B_{c}(k) y(k) \\
u(k)=C_{c}(k) x_{c}(k)
\end{array}\right.
$$

where $x_{c}(k) \in \mathbb{R}^{n_{c}}$ is the controller state, $A_{c}(k), B_{c}(k)$ and $C_{c}(k)$ are the controller parameters to be designed.

Letting $\eta(k)=\left[\begin{array}{ll}x^{T}(k) & x_{c}^{T}(k)\end{array}\right]^{T}$ and $\varpi(k)=\left[w^{T}(k)\right.$ $\left.v^{T}(k)\right]^{T}$, we have the following closed-loop system to be investigated:

$$
\left\{\begin{aligned}
& \eta(k+1)=\left(\mathcal{A}(k)+\tilde{\alpha}(k) \Delta \mathcal{A}(k)+\tilde{\mathcal{B}}_{c}(k)\right) \eta(k) \\
&+(\bar{\beta}+\tilde{\beta}(k)) \mathcal{G}(k, x(k))+\mathcal{D}(k) \varpi(k) \\
& z(k)=\mathcal{M}(k) \eta(k)
\end{aligned}\right.
$$

where

$$
\begin{aligned}
& \mathcal{A}(k)=\overline{\mathcal{A}}(k)+\Delta \overline{\mathcal{A}}(k)+\bar{\alpha} \Delta \mathcal{A}(k), \\
& \Delta \overline{\mathcal{A}}(k)=\overline{\mathcal{H}}_{a}(k) \bar{F}(k) \bar{E}_{c}(k), \tilde{\alpha}(k)=\alpha(k)-\bar{\alpha}, \\
& \Delta \mathcal{A}(k)=\mathcal{H}_{a}(k) F(k) \mathcal{N}(k), \tilde{\beta}(k)=\beta(k)-\bar{\beta}, \\
& \overline{\mathcal{A}}(k)=\left[\begin{array}{cc}
A(k) & B_{1}(k) G(k) C_{c}(k) \\
B_{c}(k) \bar{\Xi} C(k) & A_{c}(k)
\end{array}\right],
\end{aligned}
$$

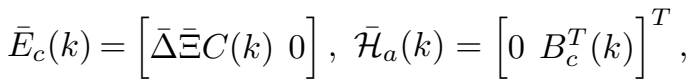

$$
\begin{aligned}
& \mathcal{H}_{a}(k)=\left[H_{a}^{T}(k) 0\right]^{T}, \tilde{\mathcal{B}}_{c}(k)=\tilde{\mathcal{B}}_{c 0}(k)+\Delta \tilde{\mathcal{B}}_{c 0}(k), \\
& \mathcal{D}(k)=\overline{\mathcal{D}}(k)+\Delta \overline{\mathcal{D}}(k), \tilde{\Xi}(k)=\Xi(k)-\bar{\Xi}, \\
& \Delta \tilde{\mathcal{B}}_{c 0}(k)=\overline{\mathcal{H}}_{a}(k) \bar{F}(k) \tilde{E}_{c}(k), \bar{E}_{E}(k)=\left[\begin{array}{ll}
0 & \bar{\Delta} \bar{E}(k)]
\end{array}\right. \text {, } \\
& \tilde{\mathcal{B}}_{c 0}(k)=\left[\begin{array}{cc}
0 & 0 \\
B_{c}(k) \tilde{\Xi}(k) C(k) & 0
\end{array}\right], \mathcal{N}(k)=[N(k) 0], \\
& \tilde{E}_{c}(k)=[\bar{\Delta} \tilde{\Xi}(k) C(k) 0] \text {, } \\
& \overline{\mathcal{D}}(k)=\operatorname{diag}\left\{D(k), B_{c}(k) \bar{E}(k)\right\}, \\
& \Delta \overline{\mathcal{D}}(k)=\overline{\mathcal{H}}_{a}(k) \bar{F}(k) \bar{E}_{E}(k), \\
& \mathcal{M}(k)=\left[M(k) B_{2}(k) G(k) C_{c}(k)\right] \text {, } \\
& \mathcal{G}(k, x(k))=\left[g^{T}(k, x(k)) 0\right]^{T} \text {. }
\end{aligned}
$$

The objective of this paper is to find a sequence of reliable controller parameters $A_{c}(k), B_{c}(k)$ and $C_{c}(k)$ such that the closed-loop system (14) satisfies the following performance requirement:

$$
\begin{aligned}
J:=\mathbb{E}\left\{\sum_{k=0}^{N-1}\left(\|z(k)\|^{2}-\gamma^{2}\|\varpi(k)\|_{R}^{2}\right)-\gamma^{2} \eta^{T}(0) S \eta(0)\right\} \\
<0
\end{aligned}
$$

where $\|\varpi(k)\|_{R}^{2}=\varpi^{T}(k) R \varpi(k)$, and $R$ and $S$ are known positive definite weighted matrices.

\section{Main results}

In this section, we investigate both the controller analysis and controller design problems for the discrete timevarying nonlinear stochastic system (1) with randomly occurring uncertainties, nonlinearities, actuator and sensor failures subject to output quantization. In the following theorem, we present the following analysis results with known parameter matrix describing the actuator failures.

Theorem 1 Consider the closed-loop system (14) with known actuator failure parameter matrix $G(k)$. Let the disturbance attenuation level $\gamma>0$, families of positive scalars $\{\varepsilon(k)\}_{0 \leq k \leq N-1}>0$, the positive definite matrices $R>0, S>0$ and the controller feedback gain matrices $\left\{A_{c}(k)\right\}_{0 \leq k \leq N-1},\left\{B_{c}(k)\right\}_{0 \leq k \leq N-1}$ and 
$\left\{C_{c}(k)\right\}_{0 \leq k \leq N-1}$ be given. The $\mathcal{H}_{\infty}$ performance requirement defined in (15) is achieved for all nonzero $\varpi(k)$ if, with the initial condition $P(0) \leq \gamma^{2} S$, there exist families of positive definite matrices $\{\bar{P}(k)\}_{0 \leq k \leq N}>0$, $\{Q(k)\}_{0 \leq k \leq N}>0$ and families of positive scalars $\{\lambda(k)>0\}_{0 \leq k \leq N-1}$ satisfying the following recursive matrix inequalities

$$
\Omega(k)=\left[\begin{array}{ccc}
\Omega_{11}(k) & * & * \\
0 & \Omega_{22}(k) & * \\
\Omega_{31}(k) & \Omega_{32}(k) & \Omega_{33}(k)
\end{array}\right]<0
$$

with the parameters updated by

$$
P(k+1)=Q^{-1}(k+1)
$$

where

$$
\begin{aligned}
& \Omega_{11}(k)=-P(k)+\lambda(k) \varepsilon(k) H^{T} E^{T}(k) E(k) H, \\
& \Omega_{22}(k)=\operatorname{diag}\left\{-\lambda(k) H_{I}^{T} H_{I},-\gamma^{2} R\right\}, H=\left[\begin{array}{ll}
I & 0
\end{array}\right] \text {, } \\
& \Omega_{31}(k)=\left[\mathcal{A}^{T}(k) \underline{B}_{c}^{T}(k) \mathcal{M}^{T}(k) \Lambda_{\alpha} \Delta \mathcal{A}^{T}(k) 0\right]^{T}, \\
& \bar{E}_{c i}(k)=\left[\begin{array}{lll}
\bar{\Delta} C_{i}(k) & 0
\end{array}\right], \Omega_{32}(k)=\left[\Omega_{321}(k) \Omega_{322}(k)\right], \\
& \Omega_{321}(k)=\left[\begin{array}{lllll}
\bar{\beta} I & 0 & 0 & 0 & \sqrt{\beta(1-\bar{\beta})} I
\end{array}\right]^{T}, H_{I}=\left[\begin{array}{ll}
I & I
\end{array}\right], \\
& \Delta \overline{\mathcal{B}}_{c i}(k)=\overline{\mathcal{H}}_{a}(k) \bar{F}(k) \bar{E}_{c i}(k), \Lambda_{\alpha}=\sqrt{\bar{\alpha}(1-\bar{\alpha})}, \\
& \Omega_{322}(k)=\left[\begin{array}{lllll}
\mathcal{D}^{T}(k) & 0 & 0 & 0 & 0
\end{array}\right]^{T}, \\
& \Omega_{33}(k)=-\operatorname{diag}\{Q(k+1), \hat{Q}(k+1), I, \widetilde{Q}(k+1)\}, \\
& Q(k+1)=P^{-1}(k+1), \hat{Q}(k+1)=I_{n_{y}} \otimes Q(k+1), \\
& \hat{\mathcal{B}}_{c i}(k)=\left[\chi_{1} \overline{\mathcal{B}}_{c_{1}}^{T}(k) \chi_{2} \overline{\mathcal{B}}_{c_{2}}^{T}(k) \cdots \chi_{n_{y}} \overline{\mathcal{B}}_{c_{n_{y}}}^{T}(k)\right]^{T}, \\
& \Delta \hat{\mathcal{B}}_{c i}(k)=\left[\begin{array}{llll}
\chi_{1} \Delta \overline{\mathcal{B}}_{c_{1}}^{T}(k) & \cdots & \chi_{n_{y}} \Delta \overline{\mathcal{B}}_{c_{n_{y}}}^{T}(k)
\end{array}\right]^{T}, \\
& \overline{\mathcal{B}}_{c i}(k)=\left[\begin{array}{cc}
0 & 0 \\
B_{c}(k) C_{i}(k) & 0
\end{array}\right], \widetilde{Q}(k+1)=I_{2} \otimes Q(k+1), \\
& \underline{B}_{c}(k)=\hat{\mathcal{B}}_{c i}(k)+\Delta \hat{\mathcal{B}}_{c i}(k) \text {. }
\end{aligned}
$$

Proof: Defining

$$
J(k)=\eta^{T}(k+1) P(k+1) \eta(k+1)-\eta^{T}(k) P(k) \eta(k),
$$

we obtain that

$$
\begin{aligned}
& \mathbb{E}\{J(k)\} \\
= & \mathbb{E}\left\{\xi^{T}(k) \bar{\Omega}(k) \xi(k)-z^{T}(k) z(k)+\gamma^{2} \varpi^{T}(k) R \varpi(k)\right\}
\end{aligned}
$$

where

$$
\begin{aligned}
& \bar{\Omega}(k)=\left[\begin{array}{ccc}
\bar{\Omega}_{11}(k) & * & * \\
\bar{\beta} P(k+1) \mathcal{A}(k) & \bar{\Omega}_{22}(k) & * \\
\bar{\Omega}_{31}(k) & \bar{\Omega}_{32}(k) & \bar{\Omega}_{33}(k)
\end{array}\right], \\
& \xi(k)=\left[\eta^{T}(k) \mathcal{G}^{T}(k, x(k)) \varpi^{T}(k)\right]^{T}, \\
& \bar{\Omega}_{22}(k)=\bar{\beta}^{2} P(k+1)+\bar{\beta}(1-\bar{\beta}) P(k+1) \text {, } \\
& \bar{\Omega}_{11}(k)=\mathcal{A}^{T}(k) P(k+1) \mathcal{A}(k)+\bar{\alpha}(1-\bar{\alpha}) \Delta \mathcal{A}^{T}(k) \\
& \times P(k+1) \Delta \mathcal{A}(k)+\left(\hat{\mathcal{B}}_{c i}(k)+\Delta \hat{\mathcal{B}}_{c i}(k)\right)^{T} \\
& \times\left(I_{n_{y}} \otimes P(k+1)\right)\left(\hat{\mathcal{B}}_{c i}(k)+\Delta \hat{\mathcal{B}}_{c i}(k)\right) \\
& +\mathcal{M}^{T}(k) \mathcal{M}(k)-P(k), \\
& \bar{\Omega}_{31}(k)=\mathcal{D}^{T}(k) P(k+1) \mathcal{A}(k), \\
& \bar{\Omega}_{32}(k)=\bar{\beta} \mathcal{D}^{T}(k) P(k+1), \\
& \bar{\Omega}_{33}(k)=\mathcal{D}^{T}(k) P(k+1) \mathcal{D}(k)-\gamma^{2} R \text {. }
\end{aligned}
$$

It follows from the constraint (2) that

$$
\left\|H_{I} \mathcal{G}(k, x(k))\right\|^{2} \leq \varepsilon(k)\|E(k) H \eta(k)\|^{2}
$$

and therefore we have

$$
\begin{aligned}
\mathbb{E}\{J(k)\} \leq & \mathbb{E}\left\{\xi^{T}(k) \bar{\Omega}(k) \xi(k)-\lambda(k)\left(\left\|H_{I} \mathcal{G}(k, x(k))\right\|^{2}\right.\right. \\
& \left.\left.-\varepsilon(k)\|E(k) H \eta(k)\|^{2}\right)\right\}-\mathbb{E}\left\{z^{T}(k) z(k)\right. \\
& \left.-\gamma^{2} \varpi^{T}(k) R \varpi(k)\right\} \\
= & \mathbb{E}\left\{\xi^{T}(k) \tilde{\Omega}(k) \xi(k)\right\}-\mathbb{E}\left\{z^{T}(k) z(k)\right. \\
& \left.-\gamma^{2} \varpi^{T}(k) R \varpi(k)\right\}
\end{aligned}
$$

where

$$
\begin{aligned}
\tilde{\Omega}(k) & =\bar{\Omega}(k)+\operatorname{diag}\left\{\lambda(k) \varepsilon(k) H_{E},-\lambda(k) H_{I}^{T} H_{I}, 0\right\}, \\
H_{E} & =H^{T} E^{T}(k) E(k) H .
\end{aligned}
$$

It follows from (16) that $\tilde{\Omega}(k)<0$. Summing up (19) on both sides from 0 to $N-1$ with respect to $k$, we obtain

$$
\begin{aligned}
& \sum_{k=0}^{N-1} \mathbb{E}\{J(k)\} \\
= & \mathbb{E}\left\{\eta^{T}(N) P(N) \eta(N)\right\}-\eta^{T}(0) P(0) \eta(0) \\
\leq & \mathbb{E}\left\{\sum_{k=0}^{N-1} \xi^{T}(k) \tilde{\Omega}(k) \xi(k)\right\}-\mathbb{E}\left\{\sum _ { k = 0 } ^ { N - 1 } \left(z^{T}(k) z(k)\right.\right. \\
& \left.\left.-\gamma^{2} \varpi^{T}(k) R \varpi(k)\right)\right\} .
\end{aligned}
$$


Hence, the $\mathcal{H}_{\infty}$ performance index defined in (15) satisfies

$$
\begin{aligned}
J \leq & \mathbb{E}\left\{\sum_{k=0}^{N-1} \xi^{T}(k) \tilde{\Omega}(k) \xi(k)\right\}-\mathbb{E}\left\{\eta^{T}(N) P(N) \eta(N)\right. \\
& \left.-\eta^{T}(0)\left(P(0)-\gamma^{2} S\right) \eta(0)\right\} .
\end{aligned}
$$

Noting that $P(N)>0, \tilde{\Omega}(k)<0$ and the initial condition $P(0) \leq \gamma^{2} S$, it can be obtained that $J<0$ and the proof is now complete.

Based on the analysis results with the desired output feedback controllers, we are now ready to solve the controller design problem for system (1) in the following theorem. For convenience of later analysis, we denote

$\Gamma_{11}(k)=\operatorname{diag}\left\{-P(k)+\lambda(k) \varepsilon(k) H_{E},-\lambda(k) H_{I}^{T} H_{I},-\gamma^{2} R\right\}$, $\overline{\mathcal{D}}_{0}(k)=\operatorname{diag}\{D(k), 0\}, D_{R}(k)=E_{I} K(k) R_{3}(k)+\overline{\mathcal{D}}_{0}(k)$, $G_{R}(k)=E_{I} K(k) R_{1}(k)+R_{2}(k) G(k) \bar{C}_{c}(k), E_{I}=\left[\begin{array}{ll}0 & I\end{array}\right]^{T}$, $\Gamma_{21}(k)=\left[\begin{array}{ccc}\overline{\mathcal{A}}_{0}(k)+G_{R}(k) & \bar{\beta} I & D_{R}(k) \\ \left(I_{n_{y}} \otimes E_{I} K(k)\right) R_{5}(k) & 0 & 0\end{array}\right]$, $\Gamma_{22}(k)=\operatorname{diag}\{-Q(k+1),-\hat{Q}(k+1)\}, R_{2}(k)=\left[\begin{array}{c}B_{1}(k) \\ 0\end{array}\right]$, $\Gamma_{31}(k)=\operatorname{diag}\left\{B_{2}(k) G(k) \bar{C}_{c}(k)+\mathcal{M}_{0}(k), \sqrt{\bar{\beta}(1-\bar{\beta})} I, 0\right\}$, $\Gamma_{33}(k)=\operatorname{diag}\{-I,-Q(k+1),-Q(k+1)\}$, $\Gamma_{42}(k)=\operatorname{diag}\left\{\left(E_{I} K(k) E_{I}\right)^{T},\left(I_{n_{y}} \otimes E_{I} K(k) E_{I}\right)^{T}\right\}$, $\Gamma_{44}(k)=\operatorname{diag}\left\{-\psi_{1}(k) I,-\psi_{1}(k) I\right\}, \mathcal{M}_{0}(k)=[M(k) 0]$, $\Gamma_{51}(k)=\left[\begin{array}{lll}\hat{E}_{a}(k) & 0 & \hat{E}_{b}(k)\end{array}\right], \Gamma_{52}(k)=\left[\begin{array}{ll}\hat{E}_{c}(k) & 0\end{array}\right]$, $\hat{E}_{a}(k)=\left[\begin{array}{lllll}\psi_{1}(k) \bar{E}_{c}^{T}(k) & \psi_{1}(k) \tilde{E}_{c i}^{T}(k) & 0 & \psi_{2}(k) \mathcal{N}^{T}(k)\end{array}\right]^{T}$, $\hat{E}_{b}(k)=\left[\begin{array}{llll}\psi_{1}(k) \bar{E}_{E}^{T}(k) & 0 & 0 & 0\end{array}\right]^{T}, \hat{E}_{c}(k)=\left[\begin{array}{llll}0 & 0 & \bar{\alpha} \mathcal{H}_{a}(k) & 0\end{array}\right]^{T}$,

$\Gamma_{55}(k)=\operatorname{diag}\left\{I_{2} \otimes\left(-\psi_{1}(k) I\right), I_{2} \otimes\left(-\psi_{2}(k) I\right)\right\}$,

$\Gamma_{53}(k)=\left[\begin{array}{lll}0 & 0 & \hat{E}_{d}(k)\end{array}\right], \hat{E}_{d}(k)=\left[\begin{array}{llll}0 & 0 & \Lambda_{\alpha} \mathcal{H}_{a}(k) & 0\end{array}\right]^{T}$,

$\overline{\mathcal{A}}_{0}(k)=\operatorname{diag}\{A(k), 0\}, R_{3}(k)=\operatorname{diag}\{0, \bar{E}(k)\}$,

$\tilde{E}_{c i}(k)=\left[\begin{array}{lllll}\chi_{1} \bar{E}_{c_{1}}^{T}(k) & \chi_{2} \bar{E}_{c_{2}}^{T}(k) & \cdots & \chi_{n_{y}} \bar{E}_{c_{n_{y}}}^{T}(k)\end{array}\right]^{T}$,

$R_{1}(k)=\left[\begin{array}{cc}0 & I \\ \Xi C(k) & 0\end{array}\right], R_{4 i}(k)=\left[\begin{array}{cc}0 & 0 \\ C_{i}(k) & 0\end{array}\right]$,

$R_{5}(k)=\left[\chi_{1} R_{41}^{T}(k) \chi_{2} R_{42}^{T}(k) \cdots \chi_{n_{y}} R_{4 n_{y}}^{T}(k)\right]^{T}$.

Theorem 2 Consider the closed-loop system (14) with known actuator failure parameter matrix $G(k)$. Let the $\left[A_{c}(k) B_{c}(k)\right]=K(k), \quad C_{c}(k)=\bar{C}_{c}(k) E_{I}$.

disturbance attenuation level $\gamma>0$, families of positive scalars $\{\varepsilon(k)\}_{0<k<N-1}>0$, the positive definite matrices $R>0$ and $S>0$ be given. The $\mathcal{H}_{\infty}$ performance requirement defined in (15) is achieved for all nonzero $\varpi(k)$ if, with the initial condition $P(0) \leq \gamma^{2} S$, there exist families of positive definite matrices $\{\bar{P}(k)\}_{0 \leq k \leq N}>0$, $\{Q(k)\}_{0 \leq k \leq N}>0$, families of real-valued matrices $\{K(k)\}_{0<k<N-1},\left\{\bar{C}_{c}(k)\right\}_{0 \leq k \leq N-1}$, families of positive scalars $\{\lambda(k)>0\}_{0 \leq k \leq N-1}, \quad\left\{\psi_{1}(k)(k)>0\right\}_{0 \leq k \leq N-1}$ and $\left\{\psi_{2}(k)>0\right\}_{0 \leq k \leq N-1}$ satisfying the following recursive matrix inequalities

$$
\Gamma(k)=\left[\begin{array}{ccccc}
\Gamma_{11}(k) & * & * & * & * \\
\Gamma_{21}(k) & \Gamma_{22}(k) & * & * & * \\
\Gamma_{31}(k) & 0 & \Gamma_{33}(k) & * & * \\
0 & \Gamma_{42}(k) & 0 & \Gamma_{44}(k) & * \\
\Gamma_{51}(k) & \Gamma_{52}(k) & \Gamma_{53}(k) & 0 & \Gamma_{55}(k)
\end{array}\right]<0
$$

with the parameters updated by

$P(k+1)=Q^{-1}(k+1)$

where the other parameters are defined in (23) and Theorem 1. Furthermore, if $\left(P(k), Q(k+1), K(k), \bar{C}_{c}(k)\right)$ is the feasible solution of (24), then the output feedback controller parameters in the form of (13) are given as follows:

Proof: To deal with the parameter uncertainties in (14), we rewrite (16) in the following form:

$$
\begin{aligned}
& \hat{\Omega}(k)+\tilde{\mathcal{M}}_{1}(k) \tilde{\mathcal{F}}(k) \tilde{\mathcal{N}}_{1}(k)+\tilde{\mathcal{N}}_{1}^{T}(k) \tilde{\mathcal{F}}^{T}(k) \tilde{\mathcal{M}}_{1}^{T}(k) \\
& +\tilde{\mathcal{M}}_{2}(k) F(k) \tilde{\mathcal{N}}_{2}(k)+\tilde{\mathcal{N}}_{2}^{T}(k) F^{T}(k) \tilde{\mathcal{M}}_{2}^{T}(k) \\
< & 0
\end{aligned}
$$


where

$$
\begin{aligned}
& \hat{\Omega}(k)=\left[\begin{array}{ccc}
\Omega_{11}(k) & * & * \\
0 & \Omega_{22}(k) & * \\
\hat{\Omega}_{31}(k) & \hat{\Omega}_{32}(k) & \Omega_{33}(k)
\end{array}\right], \\
& \tilde{\mathcal{M}}_{1}(k)=\left[\begin{array}{cccccccc}
0 & 0 & 0 & \overline{\mathcal{H}}_{a}^{T}(k) & 0 & 0 & 0 & 0 \\
0 & 0 & 0 & 0 & \left(I_{n_{y}} \otimes \overline{\mathcal{H}}_{a}(k)\right)^{T} & 0 & 0 & 0
\end{array}\right]^{T} \text {, } \\
& \hat{\Omega}_{32}(k)=\left[\Omega_{321}(k) \hat{\Omega}_{322}(k)\right], \\
& \hat{\Omega}_{322}(k)=\left[\begin{array}{lllll}
\overline{\mathcal{D}}^{T}(k) & 0 & 0 & 0 & 0
\end{array}\right]^{T} \text {, } \\
& \tilde{\mathcal{F}}(k)=\operatorname{diag}\left\{\bar{F}(k), I_{n_{y}} \otimes \bar{F}(k)\right\}, \\
& \tilde{\mathcal{N}}_{1}(k)=\left[\begin{array}{cccccccc}
\bar{E}_{c}(k) & 0 & \bar{E}_{E}(k) & 0 & 0 & 0 & 0 & 0 \\
\tilde{E}_{c i}(k) & 0 & 0 & 0 & 0 & 0 & 0 & 0
\end{array}\right],
\end{aligned}
$$

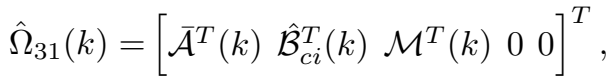

$$
\begin{aligned}
& \tilde{\mathcal{N}}_{2}(k)=\left[\begin{array}{llllllll}
\mathcal{N}(k) & 0 & 0 & 0 & 0 & 0 & 0 & 0
\end{array}\right],
\end{aligned}
$$

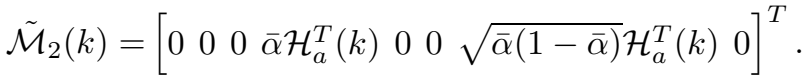

It is observed that the following inequality holds:

$$
\begin{aligned}
& {\left[\begin{array}{ccccc}
\hat{\Omega}(k) & * & * & * & * \\
\tilde{\mathcal{M}}_{1}^{T}(k) & -\psi_{1}(k) I & * & * & * \\
\psi_{1}(k) \tilde{\mathcal{N}}_{1}(k) & 0 & -\psi_{1}(k) I & * & * \\
\tilde{\mathcal{M}}_{2}^{T}(k) & 0 & 0 & -\psi_{2}(k) I & 0 \\
\psi_{2}(k) \tilde{\mathcal{N}}_{2}(k) & 0 & 0 & 0 & -\psi_{2}(k) I
\end{array}\right]} \\
& <0
\end{aligned}
$$

In order to avoid partitioning the positive define matrices $P(k)$ and $Q(k)$, the parameters in (28) are expressed as follows:

$$
\begin{aligned}
& \overline{\mathcal{A}}(k)=\overline{\mathcal{A}}_{0}(k)+E_{I} K(k) R_{1}(k)+R_{2}(k) G(k) \bar{C}_{c}(k), \\
& \overline{\mathcal{D}}(k)=\overline{\mathcal{D}}_{0}(k)+E_{I} K(k) R_{3}(k), \overline{\mathcal{H}}_{a}(k)=E_{I} K(k) E_{I}, \\
& \hat{\mathcal{B}}_{c i}(k)=\left(I_{n_{y}} \otimes E_{I} K(k)\right) R_{5}(k), \overline{\mathcal{B}}_{c i}(k)=E_{I} K(k) R_{4 i}(k) \\
& \mathcal{M}(k)=\mathcal{M}_{0}(k)+B_{2}(k) G(k) \bar{C}_{c}(k)
\end{aligned}
$$

where

$$
K(k)=\left[A_{c}(k) B_{c}(k)\right], \bar{C}_{c}(k)=\left[0 C_{c}(k)\right] .
$$

Noticing (29) and (30), (24) is obtained by (28) after applying some straightforward algebraic manipulations, and the proof of this theorem is then complete.

In Theorem 2, with known actuator failure parameter, the $\mathcal{H}_{\infty}$ performance requirement defined in (15) is obtained for the closed-loop system (14) and the output feedback controller is designed based on the recursive matrix inequalities approach. In the following theorem a design procedure for the desired controller parameters is given in the case that the failure parameter matrix $G(k)$ is unknown but satisfies the constraints (9)-(12).

Theorem 3 Consider the closed-loop system (14). Let the disturbance attenuation level $\gamma>0$, families of positive scalars $\{\varepsilon(k)\}_{0 \leq k \leq N-1}>0,\{\mu(k)\}_{0 \leq k \leq N-1}>0$, the positive definite matrices $R>0$ and $S>0$ be given. The $\mathcal{H}_{\infty}$ performance requirement defined in (15) is achieved for all nonzero $\varpi(k)$ if, with the initial condition $P(0) \leq \gamma^{2} S$, there exist families of positive definite matrices $\{P(k)\}_{0 \leq k \leq N}>0$, $\{Q(k)\}_{0 \leq k \leq N}>0$, families of real-valued matrices $\{K(k)\}_{0 \leq k \leq N-1},\left\{\bar{C}_{c}(k)\right\}_{0 \leq k \leq N-1}$, families of positive scalars $\{\lambda(k)>0\}_{0 \leq k \leq N-1}, \quad\left\{\psi_{1}(k)(k)>0\right\}_{0 \leq k \leq N-1}$ and $\left\{\psi_{2}(k)>0\right\}_{0<k<N-1}$ satisfying the following recursive matrix inequalities

$$
\begin{aligned}
\tilde{\Gamma}(k) & =\left[\begin{array}{ccc}
\hat{\Gamma}(k) & * & * \\
\mu(k) \hat{\mathcal{B}}(k) & -\mu(k) I & * \\
\hat{\mathcal{C}}_{c}(k) & 0 & -\mu(k)\left(\tilde{G}^{T}(k) \tilde{G}(k)\right)^{-1}
\end{array}\right] \\
& <0
\end{aligned}
$$

with the parameters updated by

$P(k+1)=Q^{-1}(k+1)$

where

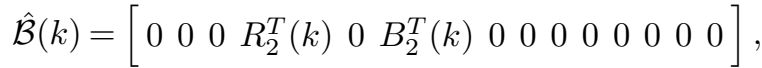

$$
\begin{aligned}
& \hat{\mathcal{C}}_{c}(k)=\left[\begin{array}{llllllllllllll}
\bar{C}_{c}(k) & 0 & 0 & 0 & 0 & 0 & 0 & 0 & 0 & 0 & 0 & 0 & 0 & 0
\end{array}\right] \text {, } \\
& \begin{aligned}
\hat{\Gamma}(k)= & {\left[\begin{array}{lcccc}
\Gamma_{11}(k) & * & * & * & * \\
\hat{\Gamma}_{21}(k) & \Gamma_{22}(k) & * & * & * \\
\hat{\Gamma}_{31}(k) & 0 & \Gamma_{33}(k) & * & * \\
0 & \Gamma_{42}(k) & 0 & \Gamma_{44}(k) & * \\
\Gamma_{51}(k) & \Gamma_{52}(k) & \Gamma_{53}(k) & 0 & \Gamma_{55}(k)
\end{array}\right], } \\
\hat{\Gamma}_{21}(k)= & {\left[\begin{array}{ccc}
\overline{\mathcal{A}}_{0}(k)+G_{0 R}(k) & \bar{\beta} I & D_{R}(k) \\
\left(I_{n_{y}} \otimes E_{I} K(k)\right) R_{5}(k) & 0 & 0
\end{array}\right], } \\
\hat{\Gamma}_{31}(k)= & \operatorname{diag}\left\{B_{G}(k), \sqrt{\bar{\beta}(1-\bar{\beta}) I, 0\}}\right. \\
B_{G}(k)= & B_{2}(k) G_{0}(k) \bar{C}_{c}(k)+\mathcal{M}_{0}(k), \\
G_{0 R}(k)= & E_{I} K(k) R_{1}(k)+R_{2}(k) G_{0}(k) \bar{C}_{c}(k) .
\end{aligned}
\end{aligned}
$$

Furthermore, if $\left(P(k), Q(k+1), K(k), \bar{C}_{c}(k)\right)$ is the feasible solution of (31), then the output feedback controller parameters in the form of (13) are given as follows:

$\left[A_{c}(k) B_{c}(k)\right]=K(k), C_{c}(k)=\bar{C}_{c}(k) E_{I}$. 
Table 1

The Reliable Controller Design Algorithm

Step 1. Give the $\mathcal{H}_{\infty}$ performance index $\gamma$, the positive definite matrices $R, S$ and the state initial condition $\eta(0)$. Select the initial value for matrix $P(0)$ which satisfy the condition $P(0) \leq \gamma^{2} S$ and set $k=0$.

Step 2. For the sampling instant $k$, solving the recursive matrix inequality (31) to obtain the values of matrix $Q(k+1)$ as well as the desired controller parameters $\left\{A_{c}(k), B_{c}(k), C_{c}(k)\right\}$.

Step 3. Set $k=k+1$ and obtain $P(k+1)$ by the parameter update formula (32).

Step 4. If $k<N$, where $N$ is the maximum number of iterations allowed, then go to Step 2, else go to Step 5.

Step 5. Stop.

Table 2

Recursive process

\begin{tabular}{|c|c|c|c|c|c|}
\hline$k$ & 0 & 1 & 2 & & $\cdots$ \\
\hline \multirow{2}{*}{$A_{c}(k)$} & 0.53140 .5314 & 0.01410 .0141 & 0.07910 .0110 & 0.31360 .3136 & \\
\hline & $\begin{array}{lll}0.5314 & 0.5314\end{array}$ & $\begin{array}{lll}0.0141 & 0.0141\end{array}$ & $\begin{array}{ll}0.0241 & 0.0698\end{array}$ & $\begin{array}{lll}0.3136 & 0.3136\end{array}$ & \\
\hline \multirow{2}{*}{$B_{c}(k)$} & $-2.0606 \quad 10.4288$ & $-0.0785 \quad 1.0544$ & $-0.0414 \quad 0.5060$ & $-0.3578 \quad 6.4767$ & \\
\hline & $-2.0606 \quad 10.4288$ & $-0.0785 \quad 1.0544$ & $-0.0414 \quad 0.5060$ & $-0.3578 \quad 6.4767$ & \\
\hline$C_{c}(k)$ & 1.87721 .8772 & $\begin{array}{lll}0.5637 & 0.5637\end{array}$ & 2.23412 .2341 & $5.2543 \quad 5.2543$ & \\
\hline
\end{tabular}

Proof: From (12), we know that $\Gamma(k)$ in Theorem 2 can be rewritten as

$\Gamma(k)=\hat{\Gamma}(k)+\hat{\mathcal{B}}^{T}(k) \Delta_{G}(k) \hat{\mathcal{C}}_{c}(k)+\hat{\mathcal{C}}_{c}^{T}(k) \Delta_{G}(k) \hat{\mathcal{B}}(k)$

Noticing inequality (12), it is obtained that

$$
\begin{aligned}
\Gamma(k) \leq & \hat{\Gamma}(k)+\mu(k) \hat{\mathcal{B}}^{T}(k) \hat{\mathcal{B}}(k)+\mu^{-1}(k) \hat{\mathcal{C}}_{c}^{T}(k) \tilde{G}^{T}(k) \\
& \times \tilde{G}(k) \hat{\mathcal{C}}_{c}(k)=\tilde{\Psi}(k) .
\end{aligned}
$$

It can be seen that (31) in Theorem 3 implies that $\Gamma(k) \leq$ $\tilde{\Psi}(k)<0$. This completes the proof.

By means of Theorem 3, the algorithm for designing the reliable robust controller can be outlined as Table 1 .

Remark 2 In the system model under investigation in this paper, there are mainly six factors that constitute the complexity and complicate the design of reliable controller, which are ROUs, RONs, actuator failure, sensor failures, quantization and time-varying parameters. It can be seen that all these six factors are explicitly reflected in our main results. In Theorem 3, the finite-horizon reliable controller is designed by solving a series of recursive linear matrix inequalities under which both the current system measurement and previous system states are employed to control the current system state. Such a recursive control process is particularly suitable for real-time implementation such as online process control.

\section{An Illustrative Example}

In this section, we present a simulation example to illustrate the effectiveness of the proposed reliable controller design scheme for discrete time-varying stochastic systems with randomly occurring uncertainties, nonlinearities, actuator and sensor failures subject to output quantization. The system data are given as follows:

$$
\begin{aligned}
A(k) & =\left[\begin{array}{cc}
-0.6 & 0.2 \\
1.1 \sin (5 k) & 0.5
\end{array}\right], H_{a}(k)=\left[\begin{array}{ll}
0.1 & 0.3
\end{array}\right]^{T}, \\
N(k) & =\left[\begin{array}{ll}
0.2 & 0
\end{array}\right], F(k)=\sin (k), B_{2}(k)=0.2, \\
B_{1}(k) & =\left[\begin{array}{c}
-2 \\
3 \sin (5 k)
\end{array}\right], D(k)=\left[\begin{array}{c}
0.1 \sin (3 k) \\
-0.3
\end{array}\right], \\
M(k) & =\left[\begin{array}{cc}
-0.4 & 0.5 \sin (5 k)
\end{array}\right], \bar{E}(k)=\left[\begin{array}{c}
0.1 \sin (3 k) \\
0.2
\end{array}\right], \\
C(k) & =\left[\begin{array}{cc}
-2+0.3 \sin (5 k) & 0.5 \\
0 & 1
\end{array}\right] .
\end{aligned}
$$

The nonlinear function $g(k, x(k))$ is selected as $g(k, x(k))=0.5 x_{1}(k) \sin \left(x_{2}(k)\right)$. It is easy to see that the constraint (2) is met with $\varepsilon(k)=1$ and $E(k)=\operatorname{diag}\{0.2,0.15\}$. The parameters of the logarithmic quantizer are chosen as $\hat{\mu}_{0}=2, \bar{\chi}_{1}=0.8$ and $\bar{\chi}_{2}=0.5$.

Let $\bar{\alpha}=0.9$ and $\bar{\beta}=0.8$. Assume that the probability density functions of $\rho_{1}(k)$ and $\rho_{2}(k)$ in $[0,1]$ are described by

$$
\zeta_{1}\left(s_{1}\right)=\left\{\begin{array}{cc}
0 & s_{1}=0 \\
0.1 & s_{1}=0.5 \\
0.9 & s_{1}=1
\end{array}, \quad \zeta_{2}\left(s_{2}\right)=\left\{\begin{array}{cc}
0 & s_{2}=0 \\
0.2 & s_{2}=0.5 \\
0.8 & s_{2}=1
\end{array}\right.\right.
$$




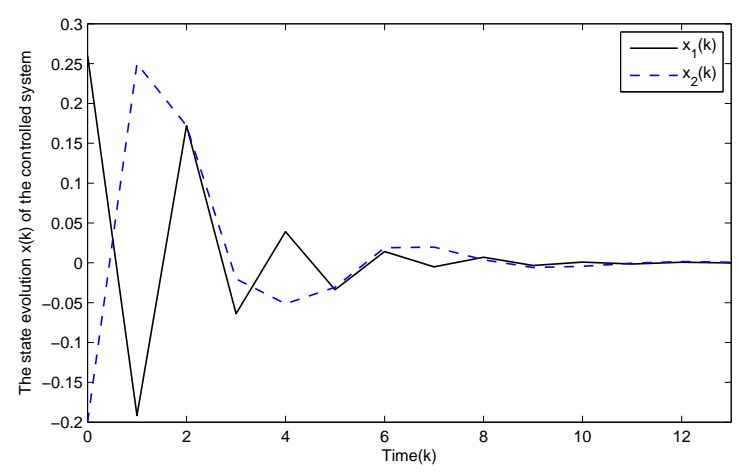

Fig. 1. The state responses of the controlled system

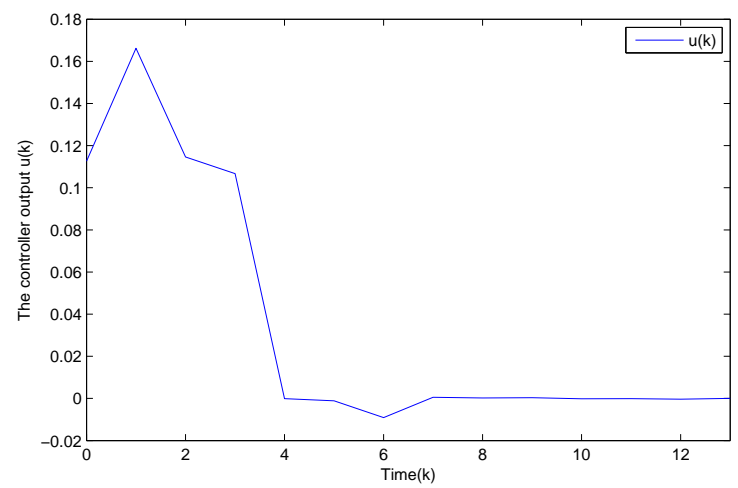

Fig. 2. The controller output $u(k)$

from which the expectations and variances can be easily calculated as $\bar{\mu}_{1}=0.95, \bar{\mu}_{2}=0.9, \chi_{1}^{2}=0.15^{2}$ and $\chi_{2}^{2}=$ 0.04 .

The actuator fault matrix $G(k)$ is assumed to satisfy $0.85 \leq G(k) \leq 0.9$. Then, we can obtain that $G_{0}(k)=$ 0.875 and $\tilde{G}(k)=0.025$. In the simulation, let $\mu(k)=1$. The $\mathcal{H}_{\infty}$ performance level is chosen as $\gamma=1$, the initial values of the states are $x_{0}=[0.26-0.2]^{T}$, $\hat{x}_{0}=[0.2-0.16]^{T}$ and the positive definite matrices $R=\operatorname{diag}\{2,2\}$ and $S=\operatorname{diag}\{2,2,2,2\}$. The exogenous disturbance input is selected as $w(k)=0.5 \sin (4 k)$ and $v(k)=0.2 \cos (4 k)$. According to reliable controller design algorithm, the desired controller parameters in Theorem 3 can be solved recursively subject to given initial conditions and prespecified performance index. Table 2 lists the desired parameters of reliable controller $A_{c}(k)$, $B_{c}(k)$ and $C_{c}(k)$ from the time $k=0$ to $k=3$.

The simulation results are shown in Figs. 1-2, where Fig. 1 plots the state simulation results of the closedloop system (14) and Fig. 2 depicts the controller output. The simulation results confirm that the desired finitehorizon performance is well achieved and the proposed reliable controller design algorithm is indeed effective.

\section{Conclusion}

In this paper, the finite-horizon reliable $\mathcal{H}_{\infty}$ outputfeedback control problem has been investigated for a class of discrete time-varying systems with ROUs, RONs as well as measurement quantizations. The actuator failures have been quantified by a variable varying in a given interval and the sensor failures have been governed by an individual random variable satisfying a certain probabilistic distribution in the interval $[0,1]$. Both the RONs and the ROUs have been modeled by the Bernoulli distributed white sequences with known conditional probabilities. In the presence of output quantization, a timevarying output feedback controller has been designed to preserve a guaranteed $\mathcal{H}_{\infty}$ performance. A simulation example has been utilized to demonstrate the effectiveness of the finite-horizon reliable control techniques presented in this paper. Other possible future research directions include real-time applications of the proposed reliable control theory in telecommunications, and further extensions of the present results to more complex systems with unreliable communication links, such as sampled data systems, bilinear systems, and a more general class of nonlinear stochastic systems.

\section{References}

[1] M. V. Basin and P. C. Rodriguez-Ramirez, Sliding mode controller design for stochastic polynomial systems with unmeasured states, IEEE Transactions on Industrial Electronics, Vol. 61, No. 1, pp. 387-396, Jan. 2014.

[2] K. Bouibed, L. Seddiki, K. Guelton and H. Akdag, Actuator and sensor fault detection and isolation of an actuated seat via nonlinear multi-observers, Systems Science and Control Engineering: An Open Access Journal, Vol. 2, No. 1, pp. 150160, 2014.

[3] R. Caballero-Aguila, A. Hermoso-Carazo and J. LinaresPerez, Covariance-based estimation algorithms in networked systems with mixed uncertainties in the observations, Signal Processing, Vol. 94, pp. 163-173, Jan. 2014.

[4] B. Chen, Y. Niu and Y. Zou, Adaptive sliding mode control for stochastic Markovian jumping systems with actuator degradation, Automatica, Vol. 49, No. 6, pp. 1748-1754, 2013.

[5] D. Ding, Z. Wang, J. Hu and H. Shu, Dissipative control for state-saturated discrete time-varying systems with randomly occurring nonlinearities and missing measurements, International Journal of Control, Vol. 86, No. 4, pp. 674-688, 2013.

[6] S. X. Ding, P. Zhang, S. Yin and E. L. Ding, An integrated design framework of fault tolerant wireless networked control systems for industrial automatic control applications, IEEE Transactions on Industrial Informatics, Vol. 9, No. 1, pp. 462-471, 2013.

[7] M. Fu and L. Xie, The sector bound approach to quantized feedback control, IEEE Transactions on Automatic Control, Vol. 50, No. 11, pp. 1698-1711, 2005.

[8] A. Gundes and H. Ozbay, Reliable decentralised control of delayed MIMO plants, International Journal of Control, Vol. 83, No. 3, pp. 516-526, 2010.

[9] M. Hernandez-Gonzalez and M. V. Basin, Discrete-time optimal control for stochastic nonlinear polynomial systems, Int. J. General Systems, Vol. 43, No. 3-4, pp. 359-371, 2014.

[10] J. Hu, Z. Wang, H. Gao and L. K. Stergioulas, Robust sliding mode control for discrete stochastic systems with mixed time delays, randomly occurring uncertainties, and randomly occurring nonlinearities, IEEE Transactions on Industrial Electronics, Vol. 59, No. 7, pp. 3008-3015, 2012. 
[11] H. R. Karimi, P. J. Maralani and B. Moshiri, Stochastic dynamic output feedback stabilization of uncertain stochastic state-delayed systems, ISA Transactions, Vol. 45, No. 2 , pp. 201-213, 2006.

[12] B. Jiang, H. Yang and P. Shi, Switching fault tolerant control design via global dissipativity, Int. J. Systems Science, Vol. 41, No. 8, pp. 1003-1012, 2010.

[13] Q. Liu, Z. Wang, X. He, and D. Zhou, A survey of eventbased strategies on control and estimation, Systems Science and Control Engineering: An Open Access Journal, Vol. 2, No. 1, pp. 90-97, 2014.

[14] Y. Liu, J. Wang and G. Yang, Reliable control of uncertain nonlinear systems, Automatica, Vol. 34, No. 7, pp. 875-879, 1998.

[15] Y.-W. Liang and S.-D. Xu, Reliable control of nonlinear systems via variable structure scheme, IEEE Transactions on Automatic Control, Vol. 51, No. 10, pp. 1721-1726, 2006.

[16] H. Ma and G. Yang, Fault-tolerant control synthesis for a class of nonlinear systems: sum of squares optimisation approach, International Journal of Robust and Nonlinear Control, Vol. 19, No. 5, pp. 591-610, 2009.

[17] Y. Niu, D. W. C. Ho and X. Wang, Robust $\mathcal{H}_{\infty}$ control for nonlinear stochastic systems: A sliding-mode approach, IEEE Transactions on Automatic Control, Vol. 53, No. 7 , pp. 1695-1701, 2008.

[18] Y. Niu, Y. Liu and T. Jia, Reliable control of stochastic systems via sliding mode technique, Optimal contro applications and methods, Vol. 34, No. 6, pp. 712-727, 2013.

[19] L. Qin, X. He and D. Zhou, A survey of fault diagnosis for swarm systems, Systems Science and Control Engineering: An Open Access Journal, Vol. 2, No. 1, pp. 13-23, 2014.

[20] B. Shen, Z. Wang and X. Liu, Sampled-data synchronization control of complex dynamical networks with stochastic sampling, IEEE Transactions on Automatic Control, Vol. 57, No. 10, pp. 2644-2650, 2012.

[21] P. Suchomski, A reliable synthesis of discrete-time $\mathcal{H}_{\infty}$ control. Part I: basic theorems and J-lossless conjugators, Control and Cybernetics, Vol. 36, No. 1, pp. 97-141, 2007.

[22] S. Tong, Y. Li and T. Wang, Adaptive fuzzy decentralized output feedback control for stochastic nonlinear large-scale systems using DSC technique, International Journal of Robust and Nonlinear Control, Vol. 23, No. 4, pp. 381-399, 2013.

[23] T. Wang, S. Tong and Y. Li, Adaptive neural network output feedback control of stochastic nonlinear systems with dynamical uncertainties, Neural Computing \& Applications, Vol. 23, No. 5, pp. 1481-1494, 2013.

[24] Z. Wang, D. W. C. Ho, Y. Liu and X. Liu, Robust $\mathcal{H}_{\infty}$ control for a class of nonlinear discrete time-delay stochastic systems with missing measurements, Automatica, Vol. 45, No. 3, pp. 684-691, 2009.

[25] Z. Wang, J. Lam, L. Ma, Y. Bo and Z. Guo, Varianceconstrained dissipative observer-based control for a class of nonlinear stochastic systems with degraded measurements, Journal of Mathematical Analysis and Applications, Vol. 377, No. 2, pp. 645-658, 2011.

[26] Z. Wang, Y. Wang and Y. Liu, Global synchronization for discrete-time stochastic complex networks with randomly occurred nonlinearities and mixed time-delays, IEEE Transactions on Neural Networks, Vol. 21, No. 1, pp. 11-25, 2010.

[27] W. Wong, E. Tian, D. Yue and R. Au, Robust reliable control for systems with random actuator fault and probabilistic nonlinearity with new characters, International Journal of Robust and Nonlinear Control, Vol. 23, No. 18, pp. 20132027, 2013.

[28] L. Wu, W. Zheng and H. Gao, Dissipativity-based sliding mode control of switched stochastic systems, IEEE Transactions on Automatic Control, Vol. 58, No. 3, pp. 785 791, 2013.
[29] G. Yang, J. Wang and Y. Soh, Reliable $\mathcal{H}_{\infty}$ controller design for linear systems, Automatica, Vol. 37, No. 5, pp. 717-725, 2001.

[30] S. Yin, S. X. Ding and H. Luo, Real-time implementation of fault tolerant control system with performance optimization, IEEE Transactions on Industrial Electronics, Vol. 61, No. 5, pp. 2402-2411, 2013.

Hongli Dong received the Ph.D. degree in Control Science and Engineering in 2012 from Harbin Institute of Technology, Harbin, China. From July 2009 to January 2010, she was a Research Assistant in the Department of Applied Mathematics, the City University of Hong Kong. From October 2010 to January 2011, she was a Research Assistant in the Department of Mechanical Engineering, the University of Hong Kong. From January 2011 to January 2012, she was a Visiting Scholar in the Department of Information Systems and Computing, Brunel University, London, U.K. From November 2012 to October 2014, she was an Alexander von Humboldt research fellow at the University of DuisburgEssen, Duisburg, Germany. She is currently a professor with the College of Electrical and Information Engineering, Northeast Petroleum University, Daqing, China.

Dr. Dong's current research interests include robust control and networked control systems. She is a very active reviewer for many international journals.

Zidong Wang was born in Jiangsu, China, in 1966. He received the B.Sc. degree in mathematics in 1986 from Suzhou University, Suzhou, China, and the M.Sc. degree in applied mathematics in 1990 and the Ph.D. degree in electrical engineering in 1994, both from Nanjing University of Science and Technology, Nanjing, China.

He is currently Professor of Dynamical Systems and Computing in the Department of Computer Science, Brunel University, U.K. From 1990 to 2002, he held teaching and research appointments in universities in China, Germany and the UK. Prof. Wang's research interests include dynamical systems, signal processing, bioinformatics, control theory and applications. He has published more than 200 papers in refereed international journals. He is a holder of the Alexander von Humboldt Research Fellowship of Germany, the JSPS Research Fellowship of Japan, William Mong Visiting Research Fellowship of Hong Kong.

Prof. Wang is a Fellow of the IEEE. He is serving or has served as an Associate Editor for 12 international journals, including IEEE Transactions on Automatic Control, IEEE Transactions on Control Systems Technology, IEEE Transactions on Neural Networks, IEEE Transactions on Signal Processing, and IEEE Transactions on Systems, Man, and Cybernetics - Systems. He is also a Fellow of the Royal Statistical Society and a member of program committee for many international conferences.

Steven X. Ding received Ph.D. degree in electrical engineering from the Gerhard-Mercator University of Duisburg, Germany, in 1992. From 1992 to 1994, he was a R\&D engineer at Rheinmetall GmbH. From 1995 to 2001, he was a professor of control engineering at the University of Applied Science Lausitz in Senftenberg, 
Germany, and served as vice president of this university during 19982000 . He is currently a professor of control engineering and the head of the Institute for Automatic Control and Complex Systems (AKS) at the University of Duisburg-Essen, Germany.

Prof. Ding's research interests are model-based and data-driven fault diagnosis, fault tolerant systems and their application in industry with a focus on automotive systems and chemical processes.

Huijun Gao received the Ph.D. degree in control science and engineering from Harbin Institute of Technology, Harbin, China, in 2005.

He was a Research Associate with the Department of Mechanical Engineering, The University of Hong Kong, from November 2003 to August 2004. From October 2005 to October 2007, he carried out his postdoctoral research with the Department of Electrical and Computer Engineering, University of Alberta, Canada. Since November 2004, he has been with Harbin Institute of Technology, where he is currently a Professor and director of the Research Institute of Intelligent Control and Systems. Dr Gao's research interests include network-based control, robust control/filter theory, time-delay systems and their engineering applications.

He is a Fellow of the IEEE, and an Associate Editor for a few international journals such as Automatica, IEEE Transactions on Industrial Electronics, IEEE Transactions on Systems Man and Cybernetics Part B: Cybernetics, IEEE Transactions on Fuzzy Systems, and IEEE Transactions on Control Systems Technology. 